\title{
Early nuclear stress testing after CABG: The new standard or too soon to tell?
}

\author{
Jared Hornberger, DO, a and Edward Hulten, MD, MPH, FACC, FASNC ${ }^{\mathrm{b}, \mathrm{c}}$ \\ a Cardiology Service, Department of Medicine, Walter Reed National Military Medical Center, \\ Bethesda, MD \\ b Evans Army Community Hospital, Fort Carson, CO \\ c Uniformed Services University of Health Sciences, Bethesda, MD
}

Received Oct 19, 2018; accepted Oct 20, 2018

doi: $10.1007 / \mathrm{s} 12350-018-01503-\mathrm{z}$

\section{See related article, pp. 1970-1978}

Coronary artery bypass grafting (CABG) offers patients with advanced coronary artery disease (CAD) a renewed opportunity for longer and healthier life. ${ }^{1}$ While much advancement in detection and treatment of coronary disease has been made since the emergence of exercise stress testing in the twentieth century, late complications following CABG still remain a concern. Graft failure occurs in up to fifteen percent of early postCABG patients, ${ }^{2,3}$ but graft failure alone does not fully explain outcomes following CABG. Indeed, the impact of graft failure has varied in different studies. ${ }^{4}$

Thus, several studies have attempted to identify post-CABG patients at increased risk for adverse clinical outcomes, although debate remains whether one diagnostic modality, if any, offers the most prognostic value. Clinical practice varies widely and is often driven by test availability and local center expertise. ${ }^{5}$ Plain exercise stress testing does offer prognostic value as seen in this study and numerous others, ${ }^{6}$ but lacks diagnostic accuracy for ischemia. Stress echocardiography post-CABG has also been associated with prognosis but has not led to outcomes benefit. A recent study of 349 post-CABG patients by Cortigiani et al demonstrated that ischemia by stress echo was associated with hazard ratio of 4.8 for future death or myocardial infarction. The same study also demonstrated that abnormal coronary flow reserve

Reprint requests: Edward Hulten, MD, MPH, FACC, FASNC, Evans Army Community Hospital, Fort Carson, CO; eddiehulten@gmail.com

J Nucl Cardiol 2020;27:1979-81.

$1071-3581 / \$ 34.00$

Copyright (C) 2018 American Society of Nuclear Cardiology.
(CFR) obtained by Doppler stress echo was associated with HR 2.3 when CFR was $\leq 2$ in the left anterior descending territory. ${ }^{7}$ However, the identification of ischemia was not shown to change management in a manner that improved prognosis. Clinicians previously routinely used SPECT MPI to screen post-CABG patients, but concern for over-utilization arose due to inability to demonstrate outcomes benefit. For example, in 2006, the ROSETTA-CABG registry compared 111 patients who underwent routine nuclear myocardial perfusion imaging(MPI) in the first year post-CABG to 284 who underwent selective testing. There was no difference by routine vs selective test strategy in coronary revascularization, myocardial infarction, or death although those undergoing routine testing had reduced invasive angiography. However, adverse clinical outcomes were uncommon and in the routine testing group occurred among those with negative screening nuclear MPI. ${ }^{8}$ Thus, screening SPECT MPI in the initial postCABG period is no longer routinely recommended. ${ }^{6}$ Although PET has improved accuracy and offers the potential for highly accurate quantitative coronary flow, there are no quality data for screening PET post-CABG and no evidence for outcomes benefit exists. Finally, advanced centers have evaluated the use of stress perfusion cardiac MRI (CMR) after PCI or CABG. For example, Bernhardt et al evaluated stress perfusion CMR in 110 patients at a mean of 423 days post-CABG and reported a $79 \%$ sensitivity and $77 \%$ specificity for graft failure compared with invasive coronary angiography but outcomes benefit data were not reported. ${ }^{9}$ Likewise, Klein et al reported a modest accuracy for stress perfusion CMR of just $77 \%$ sensitivity and $90 \%$ specificity in a smaller cohort of 78 symptomatic postCABG patients with a $63 \%$ prevalence of graft stenosis by angiography. ${ }^{10}$ This paper also did not evaluate prognosis or demonstrate an outcomes benefit to 
perfusion imaging. Routine angiography either invasively or by CTA often identifies graft failure but identification has not led to improved outcomes. ${ }^{11,12}$

In the past decades, cardiologists' understanding and use of diagnostic testing post-CABG has evolved dramatically. However, there still remains wide variation of which patients are tested and by which modality with little consensus on anatomic testing vs physiologic evaluation via stress testing alone or stress imaging. ${ }^{13-17}$ Due to the lack of contemporary data for outcomes benefit, current ACC AHA guidelines for the management of stable ischemic CAD do not support the use of routine imaging with either exercise or pharmacologic stress testing in asymptomatic patients within five years post-CABG. ${ }^{6}$ Similarly, appropriate use criteria classify the use of stress testing early after coronary revascularization as either "rarely appropriate" or "uncertain benefit.", 11,12 With the goal to improve outcomes, is there utility in routine stress testing after CABG as a method for risk assessment to identify patients who are at high risk?

In this issue of Journal of Nuclear Cardiology, Ortiz et al evaluated clinical outcomes among 87 male veterans that underwent both invasive coronary angiography as well as SPECT MPI one-year postCABG. Patients were enrolled prospectively at the Minneapolis VA Medical Center as part of the larger VA multicenter cohort study with a mean follow-up of 11 years. Although anatomic findings including graft failure by ICA did not associate with the primary clinical endpoint of heart failure or mortality, several findings from SPECT MPI did, including LVEF $\leq 45 \%$, moderate or large perfusion defect size, or inability to reach stage 3 on a standard Bruce protocol. Patients found to have $\geq 2$ of the above variables were identified to be most at risk for future adverse events.

These findings were particularly relevant when the population excluded twelve patients who underwent pharmacologic stress, consistent with practice guidelines to perform pharmacologic stress only when routine exercise testing is not possible due to co-morbidities. Exercise testing not only gives us information regarding symptoms but other variables such as chronotropic competence and exercise capacity are also evaluated that have powerful prognostic value. ${ }^{18}$

The findings add important evidence to a prevalent but understudied patient population, asymptomatic patients in the initial months to years post-CABG. However, as the investigators appropriately discussed, the sample size of 87 is underpowered to draw definitive conclusions and likely accounts for the lack of association of graft anatomy post-CABG with outcomes. Cohort characteristics did include patients with cardio- vascular risk factors such as smoking, diabetes mellitus, and chronic kidney disease; however, the study was composed solely of men and no mention was made in regard to ethnicity. Given this small sample size from a limited demographic, more prospective studies are needed at this time before any conclusion can be made regarding the utility of more frequent stress testing in asymptomatic patients. Further studies should investigate whether screening may be beneficial in certain, high-risk populations such as those who may not have achieved complete revascularization.

This helpful study by Ortiz et al draws important attention to the need to better understand how to prevent adverse clinical outcomes post-CABG and whether there may be a potential role for ischemia testing as early as one-year post-CABG for prognostication. While identifying high-risk patients is important, no study has concluded that earlier detection leads to longterm improved outcomes. Since all of the patients should be on maximal medical therapy and, in this study at least, the SPECT findings did not correlate with graft failure, it remains to be demonstrated how identifying ischemia among asymptomatic post-CABG patients would prompt a change in management that reduces morbidity and mortality, which is ultimately the goal for any diagnostic test. Furthermore, cost effectiveness studies would need to justify any call for more widespread screening among asymptomatic patients in the initial years post-CABG. So, while this study is not practice changing, the findings are certainly intriguing. Further prospective study may lead to an improved prognosis for post-CABG patients with occult high risk markers, which in this study were impaired LVEF, moderate to severe perfusion defect, and inability to complete two stages of Bruce protocol. Although a small observational study, it does add important data to an understudied but vital clinical question affecting millions of asymptomatic patients living today postCABG. Such questions are best addressed by large, randomized imaging trials comparing routine screening vs selective testing for post-CABG patients, but until such data, if ever, become available, clinicians will rely on observational data. Such studies, including the present study, will continue to offer valuable insight, and as Yogi Berra once noted, "You can observe a lot by just watching.",

\section{Disclosures}

The opinions and assertions contained herein are the authors' alone and do not represent the views of Walter Reed National Military Medical Center, Evans Army Community Hospital, the US Army, or the Department of Defense. 


\section{References}

1. Hillis LD, Smith PK, Anderson JL, Bittl JA, Bridges CR, Byrne JG, et al. 2011 ACCF/AHA guideline for coronary artery bypass graft surgery: a report of the American College of Cardiology Foundation/American Heart Association Task Force on Practice Guidelines developed in collaboration with the American Association for Thoracic Surgery, Society of Cardiovascular Anesthesiologists, and Society of Thoracic Surgeons. J Am Coll Cardiol 2011;58:e123-210.

2. Goldman S, Zadina K, Moritz T, Ovitt T, Sethi G, Copeland JG, et al. Long-term patency of saphenous vein and left internal mammary artery grafts after coronary artery bypass surgery: Results from a Department of Veterans Affairs Cooperative Study. J Am Coll Cardiol 2004;44:2149-56.

3. Goldman S, Sethi GK, Holman W, Thai H, McFalls E, Ward HB, et al. Radial artery grafts vs saphenous vein grafts in coronary artery bypass surgery: A randomized trial. Jama 2011;305:167-74.

4. Lopes RD, Mehta RH, Hafley GE, Williams JB, Mack MJ, Peterson ED, et al. Relationship between vein graft failure and subsequent clinical outcomes after coronary artery bypass surgery. Circulation 2012;125:749-56.

5. Eisenberg MJ, Wou K, Nguyen H, Duerr R, Del Core M, Fourchy $\mathrm{D}$, et al. Use of stress testing early after coronary artery bypass graft surgery. Am J Cardiol 2006;97:810-6.

6. Fihn SD, Gardin JM, Abrams J, Berra K, Blankenship JC, Douglas PS, et al. 2012 ACCF/AHA/ACP/AATS/PCNA/SCAI/STS guideline for the diagnosis and management of patients with stable ischemic heart disease: a report of the American College of Cardiology Foundation/American Heart Association task force on practice guidelines, and the American College of Physicians, American Association for Thoracic Surgery, Preventive Cardiovascular Nurses Association, Society for Cardiovascular Angiography and Interventions, and Society of Thoracic Surgeons. J Am Coll Cardiol 2012;60:e44-164.

7. Cortigiani L, Ciampi Q, Rigo F, Bovenzi F, Picano E, Sicari R. Prognostic value of dual imaging stress echocardiography following coronary bypass surgery. Int J Cardiol 2018.

8. Eisenberg MJ, Wou K, Nguyen H, Duerr R, Del Core M, Fourchy $\mathrm{D}$, et al. Lack of benefit for routine functional testing early after coronary artery bypass graft surgery: Results from the ROSETTACABG Registry. J Invasive Cardiol 2006;18:147-52.

9. Bernhardt P, Spiess J, Levenson B, Pilz G, Höfling B, Hombach V, et al. Combined assessment of myocardial perfusion and late gadolinium enhancement in patients after percutaneous coronary intervention or bypass grafts: A multicenter study of an integrated cardiovascular magnetic resonance protocol. JACC 2009;2:1292300 .
10. Klein C, Nagel E, Gebker R, Kelle S, Schnackenburg B, Graf K, et al. Magnetic resonance adenosine perfusion imaging in patients after coronary artery bypass graft surgery. JACC Cardiovasc Imaging 2009;2:437-45.

11. Hendel R, Berman D, Di Carli M, Heidenreich P, Henkin R, Pellikka P. Appropriate use criteria for cardiac radionuclide imaging: A report of the American College of Cardiology Foundation Appropriate Use Criteria Task Force, the American Society of Nuclear Cardiology, the American College of Radiology, the American Heart Association, the American Society of Echocardiography, the Society of Cardiovascular Computed Tomography, the Society for Cardiovascular Magnetic Resonance, and the Society of Nuclear Medicine. J Am Coll Cardiol 2009;53:2201-29.

12. Wolk M, Bailey S, Doherty J, Douglas P, Hendel R, Kramer C, et al. Multimodality appropriate use criteria for the detection and risk assessment of stable ischemic heart disease: A report of the American College of Cardiology Foundation Appropriate Use Criteria Task Force, American Heart Association, American Society of Echocardiography, American Society of Nuclear Cardiology. Heart Fail Soc Am Heart Rhythm Soc Soc Cardiovasc Angiogr Interventions Soc Cardiovasc Comput Tomogr Soc Cardiovasc Magn Reson Soc Thorac Surg J Am Coll Cardiol 2014;63:380-406.

13. Shaw LJ, Cerqueira MD, Brooks MM, Althouse AD, Sansing VV, Beller GA, et al. Impact of left ventricular function and the extent of ischemia and scar by stress myocardial perfusion imaging on prognosis and therapeutic risk reduction in diabetic patients with coronary artery disease: Results from the Bypass Angioplasty Revascularization Investigation 2 Diabetes (BARI 2D) trial. J Nucl Cardiol 2012;19:658-69.

14. Lauer MS, Lytle B, Pashkow F, Snader CE, Marwick TH. Prediction of death and myocardial infarction by screening with exercise-thallium testing after coronary-artery-bypass grafting. Lancet 1998;351:615-22.

15. Mushtaq S, Andreini D, Pontone G, Bertella E, Bartorelli AL, Conte E, et al. Prognostic value of coronary CTA in coronary bypass patients: A long-term follow-up study. JACC 2014;7:5809.

16. Weustink AC, Nieman K, Pugliese F, Mollet NR, Meijboom BW, van Mieghem C, et al. Diagnostic accuracy of computed tomography angiography in patients after bypass grafting: Comparison with invasive coronary angiography. JACC 2009;2:816-24.

17. Chow BJ, Ahmed O, Small G, Alghamdi A-A, Yam Y, Chen L, et al. Prognostic value of $\mathrm{CT}$ angiography in coronary bypass patients. JACC 2011;4:496-502.

18. Myers J, Prakash M, Froelicher V, Do D, Partington S, Atwood JE. Exercise capacity and mortality among men referred for exercise testing. N Engl J Med 2002;346:793-801. 